\title{
Association Between Choice of Radical Prostatectomy, External Beam Radiotherapy, Brachytherapy, or Active Surveillance and Patient-Reported Quality of Life Among Men With Localized Prostate Cancer
}

\author{
Ronald C. Chen, MD, MPH; Ramsankar Basak, PhD; Anne-Marie Meyer, PhD; Tzy-Mey Kuo, PhD; William R. Carpenter, PhD; \\ Robert P. Agans, PhD; James R. Broughman, BS; Bryce B. Reeve, PhD; Matthew E. Nielsen, MD, MS; Deborah S. Usinger, BA; \\ Kiayni C. Spearman, BS; Sarah Walden, BA; Dianne Kaleel, BA; Mary Anderson, MPH; Til Stürmer, MD, PhD; Paul A. Godley, MD, PhD
}

IMPORTANCE Patients diagnosed with localized prostate cancer have to decide among treatment strategies that may differ in their likelihood of adverse effects.

OBJECTIVE To compare quality of life (QOL) after radical prostatectomy, external beam radiotherapy, and brachytherapy vs active surveillance.

DESIGN, SETTING, AND PARTICIPANTS Population-based prospective cohort of 1141 men (57\% participation among eligible men) with newly diagnosed prostate cancer were enrolled from January 2011 through June 2013 in collaboration with the North Carolina Central Cancer Registry. Median time from diagnosis to enrollment was 5 weeks, and all men were enrolled with written informed consent prior to treatment. Final follow-up date for current analysis was September 9, 2015.

EXPOSURES Treatment with radical prostatectomy, external beam radiotherapy, brachytherapy, or active surveillance.

MAIN OUTCOMES AND MEASURES Quality of life using the validated instrument Prostate Cancer Symptom Indices was assessed at baseline (pretreatment) and 3, 12, and 24 months after treatment. The instrument contains 4 domains-sexual dysfunction, urinary obstruction and irritation, urinary incontinence, and bowel problems-each scored from 0 (no dysfunction) to 100 (maximum dysfunction). Propensity-weighted mean domain scores were compared between each treatment group vs active surveillance at each time point.

RESULTS Of 1141 enrolled men, 314 pursued active surveillance (27.5\%), 469 radical prostatectomy (41.1\%), 249 external beam radiotherapy (21.8\%), and 109 brachytherapy (9.6\%). After propensity weighting, median age was 66 to 67 years across groups, and $77 \%$ to $80 \%$ of participants were white. Across groups, propensity-weighted mean baseline scores were 41.8 to 46.4 for sexual dysfunction, 20.8 to 22.8 for urinary obstruction and irritation, 9.7 to 10.5 for urinary incontinence, and 5.7 to 6.1 for bowel problems. Compared with active surveillance, mean sexual dysfunction scores worsened by 3 months for patients who received radical prostatectomy $(36.2$ [95\% Cl, 30.4-42.0]), external beam radiotherapy (13.9 [95\% Cl, 6.7-21.2]), and brachytherapy (17.1 [95\% Cl, 7.8-26.6]). Compared with active surveillance at 3 months, worsened urinary incontinence was associated with radical prostatectomy (33.6 [95\% Cl, 27.8-39.2]); acute worsening of urinary obstruction and irritation with external beam radiotherapy $(11.7$ [95\% Cl, 8.7-14.8]) and brachytherapy (20.5 [95\% Cl, 15.1-25.9]); and worsened bowel symptoms with external beam radiotherapy (4.9 [95\% Cl, 2.4-7.4]). By 24 months, mean scores between treatment groups vs active surveillance were not significantly different in most domains.

CONCLUSIONS AND RELEVANCE In this cohort of men with localized prostate cancer, each treatment strategy was associated with distinct patterns of adverse effects over 2 years. These findings can be used to promote treatment decisions that incorporate individual preferences.

JAMA. 2017;317(11):1141-1150. doi:10.1001/jama.2017.1652
Editorial page 1121

Related article page 1126

Supplemental content

CME Quiz at

jamanetworkcme.com
Author Affiliations: Author affiliations are listed at the end of this article.

Corresponding Author: Ronald C Chen, MD, MPH, Department of Radiation Oncology, University of North Carolina at Chapel Hill, CB \#7512, 101 Manning Dr, Chapel Hill, NC 27599 (ronald_chen@med.unc.edu). 
$\mathrm{T}$ he comparative effectiveness of contemporary treatment options for localized prostate cancer is one of the highest priority research questions according to the National Academy of Medicine (previously the Institute of Medicine). ${ }^{1}$ Because patients with prostate cancer often have extended life expectancy, the quality-of-life (QOL) effects of different treatment options is a central consideration for many men in their decision-making process.

Since the mid-2000s, technologic advances in surgical and radiation treatments-eg, robotic prostatectomy and intensity-modulated radiotherapy-have been rapidly disseminated in clinical practice. ${ }^{2,3}$ These new technologies were developed with a primary purpose to reduce treatmentrelated morbidity rather than improve cure. Studies showed that surgery and radiotherapy can cause sexual, urinary, and bowel dysfunction ${ }^{4}$; however, it is unclear whether contemporary technologies cause the same magnitude of problems. In addition, with increasing recognition that many patients may not need treatment immediately, active surveillance is now a standard option, ${ }^{5}$ which preserves QOL for an unknown period for each man, until treatment is needed at the time of cancer progression.

The North Carolina Prostate Cancer Comparative Effectiveness \& Survivorship Study (NC ProCESS) is a populationbased, observational cohort of men with newly diagnosed prostate cancer who were all enrolled prior to treatment and followed prospectively. The purpose of this study was to analyze QOL changes from baseline (pretreatment) through 2 years after treatment for men who received radical prostatectomy, external beam radiotherapy, or brachytherapy vs those who pursued active surveillance.

\section{Methods}

This study was approved by the University of North Carolina institutional review board. Patients were recruited in collaboration with the Rapid Case Ascertainment system of the North Carolina Central Cancer Registry (NCCCR). All participants signed written informed consent. The NCCCR achieves the highest North American Association of Central Cancer Registries standard for complete and accurate cancer incidence data (gold certificate), with more than 95\% data completeness and $100 \%$ error-free data on cancer type, sex, race, and age. NCCCR does not collect data on adverse effects. Rapid Case Ascertainment is an accelerated incident reporting process in which registry staff proactively obtain information regarding tumors relevant to the study from local hospitals and facilities throughout all 100 counties of the state. This system allowed completion of baseline survey a median of 5 weeks after diagnosis.

\section{Data Collection}

Demographics-age, race, insurance, education, household income, marital status-were assessed by patient report on the baseline survey. Race was collected in fixed categories to assess diversity of the cohort and also used as part of propensity score calculations to adjust for potential differences

\section{Key Points}

Question What differences in quality of life are associated with radical prostatectomy, external beam radiotherapy, brachytherapy, and active surveillance after treatment for prostate cancer?

Findings In this population-based, prospective cohort of 1141 men diagnosed from January 2011 through June 2013, radical prostatectomy was associated with worse sexual dysfunction and urinary incontinence compared with active surveillance, external beam radiotherapy and brachytherapy with worse short-term urinary obstruction and irritation, and external beam radiotherapy with worse short-term bowel symptoms. However, by 24 months, mean scores between treatment groups vs active surveillance were not significantly different in most domains.

Meaning Contemporary treatment strategies were associated with distinct patterns of adverse effects over 2 years.

across comparison groups. Treatments received and dates were determined by medical record abstraction; if a medical record was unavailable, then cancer registry data were used. Because the primary goal of this study was to inform patients' initial treatment decision making, treatment group assignment for data analysis was based on the initial treatment received (eg, men who first received a prostatectomy and subsequently received an additional salvage treatment were categorized in the prostatectomy group). Active surveillance was defined with medical records stating this as the plan.

\section{Outcomes}

QOL was assessed using the validated Prostate Cancer Symptom Indices (PCSI) ${ }^{6}$; all surveys were conducted by telephone similar to prior methodology, ${ }^{7}$ at baseline (pretreatment) and 3, 12, and 24 months after the treatment date. For active surveillance, an anchor date was assigned as 3 months after diagnosis for purposes of calculating subsequent survey dates. Each participant at each time point was called up to 10 times over a 3-week period (including evening and weekend times) to maximize response. Date of the last survey used in the current analysis was September 9, 2015.

The PCSI has 4 domains: urinary obstruction and irritation (5 items), urinary incontinence (3 items), sexual dysfunction ( 5 items), and bowel problems (6 items). Each domain is scored from 0 to 100, with a higher score indicating more or worse dysfunction. Minimal clinically important differences for PCSI domains have not been defined. Parallel to each of the 4 domains are additional questions which ask patients about the magnitude of bother related to urinary obstruction and irritation, urinary incontinence, sexual, and bowel symptoms. In addition to numerical scoring for each domain described above, the PCSI has established functional levels (normal, intermediate, and poor) that incorporate the symptom as well as bother questions. ${ }^{8,9}$ These function levels complement the score reporting because QOL score changes are well recognized to be difficult for patients and physicians to interpret. ${ }^{10}$ Normal function describes a patient with essentially no dysfunction or distress in a domain ${ }^{11,12}$ 
(eg, normal function in the urinary incontinence domain describes a man who has full urinary control without incontinence). Intermediate function describes a patient with at least 1 distressful symptom but none very distressful (eg, intermediate urinary incontinence represents leaking only at certain times, and no more than a few drops). Poor function describes patients with at least 1 very distressful symptom (eg, poor sexual function represents no erections capable of intercourse or a lot of difficulty getting and keeping an erection). This classification adds clinical meaning to numerical scores, and also allowed analysis of QOL outcomes stratified by each participant's baseline level. ${ }^{9}$ Effect sizes between functional levels of the PCSI are large..$^{9,12}$

\section{Data Analysis}

To adjust for potentially important differences in baseline characteristics across cohorts, propensity scores were estimated separately for each of the 3 treatment types (external beam radiotherapy, brachytherapy, and prostatectomy) vs active surveillance. Propensity scores were obtained from the predicted probabilities in separate logistic regression models contrasting active surveillance against each of the 3 treatment types. Variables included in the regression models were race, insurance, marital status, education, income, year of diagnosis, age at diagnosis, and baseline 12-Item Short Form Survey (SF-12) and PCSI domain scores. Using the propensity scores, all patients in the 3 treatment groups were assigned weights from their propensity scores odds [propensity scores / ( 1 - propensity scores)], whereas patients in active surveillance each received a weight of $1 .^{13}$ This methodology balances the observed covariate distribution between the treatment groups relative to active surveillance allowing comparison of each treatment group's QOL outcomes against active surveillance. ${ }^{14}$ Standardized differences ${ }^{15}$ were calculated to compare the distributions of covariates between the cohorts both before and after propensity score-weighting to assess that the covariate balancing adequately addressed measured confounding across cohorts.

Propensity-weighted QOL scores for each PCSI domain was calculated for each participant at each time point. Missing data at 3,12, and 24 months were multiply imputed using the fully conditional specification approach. ${ }^{13,16}$ Multiple imputation was separately run for each of 4 domains within each treatment group for men with nonmissing data at baseline. This method sequentially regressed an outcome on the immediately preceding outcome (time point) score and baseline characteristics and replaced the missing values with values from observed (nonmissing) data that were closest to the modelpredicted value for the respective observation. To account for potential nonindependence due to weighting, robust standard errors were used in the computation of $95 \%$ CIs from the imputed data sets. To account for uncertainty occurring due to imputation, final estimates were derived by pooling estimates from all imputed data sets. Missing QOL functional levels were also imputed using a similar approach. Ordinal logistic regression was used to impute missing levels using the preceding time point functional level and QOL score and baseline characteristics as variables.
The primary goal of this study was to compare the domain scores of each treatment group (external beam radiotherapy, brachytherapy, prostatectomy) with those of active surveillance. We used an established convention ${ }^{17-19}$ to denote clinically meaningful differences in scores as those which exceed 0.5 standard deviation of the baseline score of the active surveillance (control) group. In addition to analyses of the overall cohort, stratified subgroup analyses based on patients' baseline QOL level in each domain were performed to assess if treatments differentially affected men who had different levels of baseline QOL.

All tests had a 2-sided $P$ value of less than .05 for statistical significance. Analyses were performed using SAS (SAS Institute), version 9.4.

\section{Results}

From January 2011 through June 2013, 1419 men with localized prostate cancer who had not yet started treatment were enrolled; this represents $57 \%$ participation among eligible men. ${ }^{20}$ Participants vs nonparticipants were younger (median age, 65 years vs 68 years), with nonsignificantly different Gleason scores and race. ${ }^{20}$ Among enrolled men, 69 did not receive any of the 4 modalities to be compared in this analysis, and an additional 209 did not complete any follow-up survey. Therefore, this analysis included a final sample of 1141 men.

Among 1141 men, 314 received active surveillance (27.5\%), 249 external beam radiotherapy (21.8\%), 109 brachytherapy (9.6\%), and 469 prostatectomy (41.1\%) (Table 1). Among those who received external beam radiotherapy, $94.8 \%$ received intensity-modulated radiotherapy, and 70.7\% had image guidance. Among those who received radical prostatectomy, 86.6\% received robotic surgery. Participants were sociodemographically diverse in race, education, and income. Patients who received prostatectomy were younger and had better baseline QOL than patients who received radiotherapy. Differences in baseline demographic characteristics and QOL were minimized with propensity weighting. After propensity weighting, median age was 66 to 67 years across groups, and $77 \%$ to $80 \%$ of participants were white.

Missing data in QOL are detailed in eTable 1 in the Supplement. At 24 months, there was missing data in 19\% to $29 \%$ of participants across groups and QOL domains.

\section{Sexual Dysfunction}

Propensity-weighted mean domain scores across the 4 groups at each time point were summarized in Table 2 . Sensitivity analysis, which included the 209 patients with baseline but no follow-up survey data, are shown in eTable 2 in the Supplement. At baseline, propensity-weighted scores were 43.4 (95\% CI, 39.2-47.6) for active surveillance, 41.8 (95\% CI, 36.3-47.2) for external beam radiotherapy, 46.4 (95\% CI, 36.156.7) for brachytherapy, and 41.6 (95\% CI, 35.6-47.6) for prostatectomy. Mean scores for the active surveillance group increased gradually over time. At 3 and 12 months but not 24 months, patients who received external beam radiotherapy 


\begin{tabular}{|c|c|c|c|c|c|c|c|c|}
\hline & \multicolumn{4}{|c|}{ Before Propensity Weighting, №. (\%) } & \multicolumn{4}{|c|}{ After Propensity Weighting, $\%$} \\
\hline & $\begin{array}{l}\text { Active } \\
\text { Surveillance } \\
(\mathrm{n}=314)\end{array}$ & $\begin{array}{l}\text { External } \\
\text { Beam RT } \\
(\mathrm{n}=249)\end{array}$ & $\begin{array}{l}\text { Brachytherapy } \\
(n=109)\end{array}$ & $\begin{array}{l}\text { Radical } \\
\text { Prostatectomy } \\
(\mathrm{n}=469)\end{array}$ & $\begin{array}{l}\text { Active } \\
\text { Surveillance }^{\mathrm{a}}\end{array}$ & $\begin{array}{l}\text { External } \\
\text { Beam RT }\end{array}$ & Brachytherapy & $\begin{array}{l}\text { Radical } \\
\text { Prostatectomy }\end{array}$ \\
\hline $\begin{array}{l}\text { Age at diagnosis, } \\
\text { mean (SD), y }\end{array}$ & $67(7.3)$ & $67(7.1)$ & $66(7.3)$ & $62(6.8)$ & $67(7.3)$ & $67(7.3)$ & $67(7.6)$ & $66(7.3)$ \\
\hline \multicolumn{9}{|l|}{ Race } \\
\hline White & $242(77)$ & $154(62)$ & $82(75)$ & $347(74)$ & 77 & 79 & 77 & 80 \\
\hline African American & $69(22)$ & $84(34)$ & $26(24)$ & $111(24)$ & 22 & 19 & 20 & 19 \\
\hline Other & $3(1)$ & $11(4)$ & $1(1)$ & $11(2)$ & 1 & 2 & 3 & 2 \\
\hline \multicolumn{9}{|l|}{ Health insurance } \\
\hline Medicare & $156(50)$ & $134(54)$ & $58(53)$ & $139(30)$ & 50 & 50 & 51 & 51 \\
\hline Private & $94(30)$ & $61(25)$ & $31(28)$ & $246(53)$ & 30 & 30 & 28 & 30 \\
\hline Medicaid/None & $64(20)$ & $54(22)$ & $20(18)$ & $84(18)$ & 20 & 20 & 21 & 19 \\
\hline \multicolumn{9}{|l|}{ Education } \\
\hline$\leq$ High school & $93(30)$ & $99(40)$ & $42(38)$ & $131(28)$ & 30 & 28 & 29 & 29 \\
\hline Some college & $86(27)$ & $71(29)$ & $35(32)$ & $138(29)$ & 27 & 29 & 27 & 27 \\
\hline College graduate & $135(43)$ & $79(32)$ & $32(29)$ & $200(43)$ & 43 & 43 & 44 & 44 \\
\hline \multicolumn{9}{|c|}{ Household income, $\$$ yearly } \\
\hline$<40000$ & $117(37)$ & $128(51)$ & $51(47)$ & $139(30)$ & 37 & 35 & 36 & 34 \\
\hline $40000-70000$ & $88(28)$ & $64(26)$ & $35(32)$ & $129(28)$ & 28 & 29 & 32 & 27 \\
\hline$>70000-90000$ & $44(14)$ & $23(9)$ & $13(12)$ & $69(15)$ & 14 & 15 & 13 & 15 \\
\hline$>90000$ & $65(21)$ & $34(14)$ & $10(9)$ & $132(28)$ & 21 & 20 & 19 & 23 \\
\hline Married & $257(82)$ & $184(74)$ & $93(85)$ & $372(79)$ & 82 & 82 & 79 & 78 \\
\hline
\end{tabular}

Baseline QOL Scores, Mean (SD)

$\mathrm{SF}-12^{\mathrm{b}}$

\begin{tabular}{|c|c|c|c|c|c|c|c|c|}
\hline Physical & 48.7 (10.6) & 48.1 (11.0) & $49.9(9.6)$ & $51.2(9.8)$ & $48.7(10.6)$ & $48.8(11.2)$ & $48.9(9.7)$ & 49.7 (9.9) \\
\hline Mental & $55.2(7.7)$ & $53.1(9.7)$ & $54.7(8.2)$ & $52.2(9.5)$ & $55.2(7.7)$ & $55.4(7.9)$ & $55.5(7.9)$ & $55.5(7.9)$ \\
\hline \multicolumn{9}{|l|}{ PCSI ${ }^{\mathrm{C}}$} \\
\hline Sexual dysfunction & $43.4(37.6)$ & $51.1(39.5)$ & $46.5(38.7)$ & $35.6(36.6)$ & $43.4(37.6)$ & $41.8(39.5)$ & $46.4(40.1)$ & $41.6(38.0)$ \\
\hline $\begin{array}{l}\text { Urinary obstruction } \\
\text { and irritation }\end{array}$ & $22.8(13.5)$ & $23.6(15.5)$ & $17.7(10.2)$ & $22.9(14.2)$ & $22.8(13.5)$ & $22.3(14.8)$ & $20.8(10.5)$ & $22.6(13.6)$ \\
\hline Urinary incontinence & $10.4(20.4)$ & $13.0(22.1)$ & $6.2(14.8)$ & 7.9 (16.7) & $10.4(20.4)$ & 9.9 (19.7) & $10.5(20.8)$ & $9.7(18.4)$ \\
\hline Bowel problems & $5.9(8.0)$ & $7.2(10.7)$ & $5.0(7.6)$ & $5.8(8.4)$ & $5.9(8.0)$ & $5.7(8.5)$ & $6.1(7.5)$ & $6.2(8.0)$ \\
\hline \multicolumn{9}{|l|}{$\begin{array}{l}\text { Patients with normal } \\
\text { baseline PCSI function }\end{array}$} \\
\hline Sexual dysfunction & $90(29.0)$ & $54(21.8)$ & $26(24.5)$ & 167 (35.9) & 29.0 & 31.3 & 27.1 & 28.1 \\
\hline $\begin{array}{l}\text { Urinary obstruction } \\
\text { and irritation }\end{array}$ & $68(22.3)$ & $58(23.5)$ & $36(34.3)$ & $125(27.1)$ & 22.3 & 25.9 & 30.7 & 24.1 \\
\hline Urinary incontinence & $226(73.9)$ & $169(69.3)$ & $86(81.9)$ & $360(78.1)$ & 73.9 & 76.4 & 75.6 & 74.8 \\
\hline Bowel problems & $134(43.2)$ & $99(40.1)$ & $52(49.1)$ & 205 (44.3) & 43.2 & 44.3 & 36.6 & 42.4 \\
\hline
\end{tabular}

\begin{tabular}{|c|c|c|c|c|c|c|c|c|}
\hline \multicolumn{9}{|c|}{ Variables Not Included in Propensity Score Calculation } \\
\hline \multicolumn{9}{|c|}{$\mathrm{PSA}, \mathrm{ng} / \mathrm{mL}$} \\
\hline $0-9.9$ & $273(89.2)$ & $184(74.8)$ & $97(90.7)$ & $407(87.2)$ & 89.2 & 80.1 & 91.8 & 87.3 \\
\hline $10-20$ & $19(6.2)$ & $35(14.2)$ & $8(7.5)$ & $40(8.6)$ & 6.2 & 11.9 & 6.5 & 7.2 \\
\hline$>20$ & $14(4.6)$ & $27(11.0)$ & $2(1.9)$ & $20(4.3)$ & 4.6 & 8.0 & 1.7 & 5.5 \\
\hline \multicolumn{9}{|c|}{ Biopsy Gleason score } \\
\hline$\leq 6$ & $268(86.2)$ & $88(35.8)$ & $84(79.2)$ & $213(45.6)$ & 86.2 & 39.2 & 72.7 & 46.8 \\
\hline 7 & $41(13.2)$ & $121(49.2)$ & $21(19.8)$ & 207 (44.3) & 13.2 & 46.5 & 23.1 & 41.6 \\
\hline $8-10$ & $2(0.6)$ & $37(15.0)$ & $1(0.9)$ & $47(10.1)$ & 0.6 & 14.4 & 4.2 & 11.6 \\
\hline \multicolumn{9}{|c|}{ Clinical stage } \\
\hline T1 & $276(88.7)$ & $179(72.8)$ & 94 (87.9) & $348(74.4)$ & 88.8 & 73.2 & 79.2 & 71.8 \\
\hline $\mathrm{T} 2$ & 35 (11.3) & $61(24.8)$ & $13(12.1)$ & $114(24.4)$ & 11.3 & 24.9 & 20.8 & 26.8 \\
\hline $\mathrm{T} 3$ or $\mathrm{T} 4$ & 0 & $6(2.4)$ & 0 & $6(1.3)$ & 0 & 1.9 & 0 & 1.5 \\
\hline
\end{tabular}

Abbreviations: IQR, interquartile range; PCSI, prostate cancer symptom indices; PSA, prostate-specific antigen; QOL, quality of life; $R T$, radiotherapy.

a Propensity weight for active surveillance was set as 1.

bSF-12 Mental and Physical Function: scores range 0-100, with higher score indicating better quality of life.

c PCSI domain ${ }^{6}$ scores range 0-100, with higher score indicating more symptoms and dysfunction. Minimally clinically important difference not defined for this instrument. 
Table 2. Propensity-Weighted Prostate Cancer Symptom Indices Scores for Sexual, Urinary, and Bowel Symptoms Over Time Across Different Treatment Groups Among Men With Newly Diagnosed Prostate Cancer ${ }^{\mathrm{a}}$

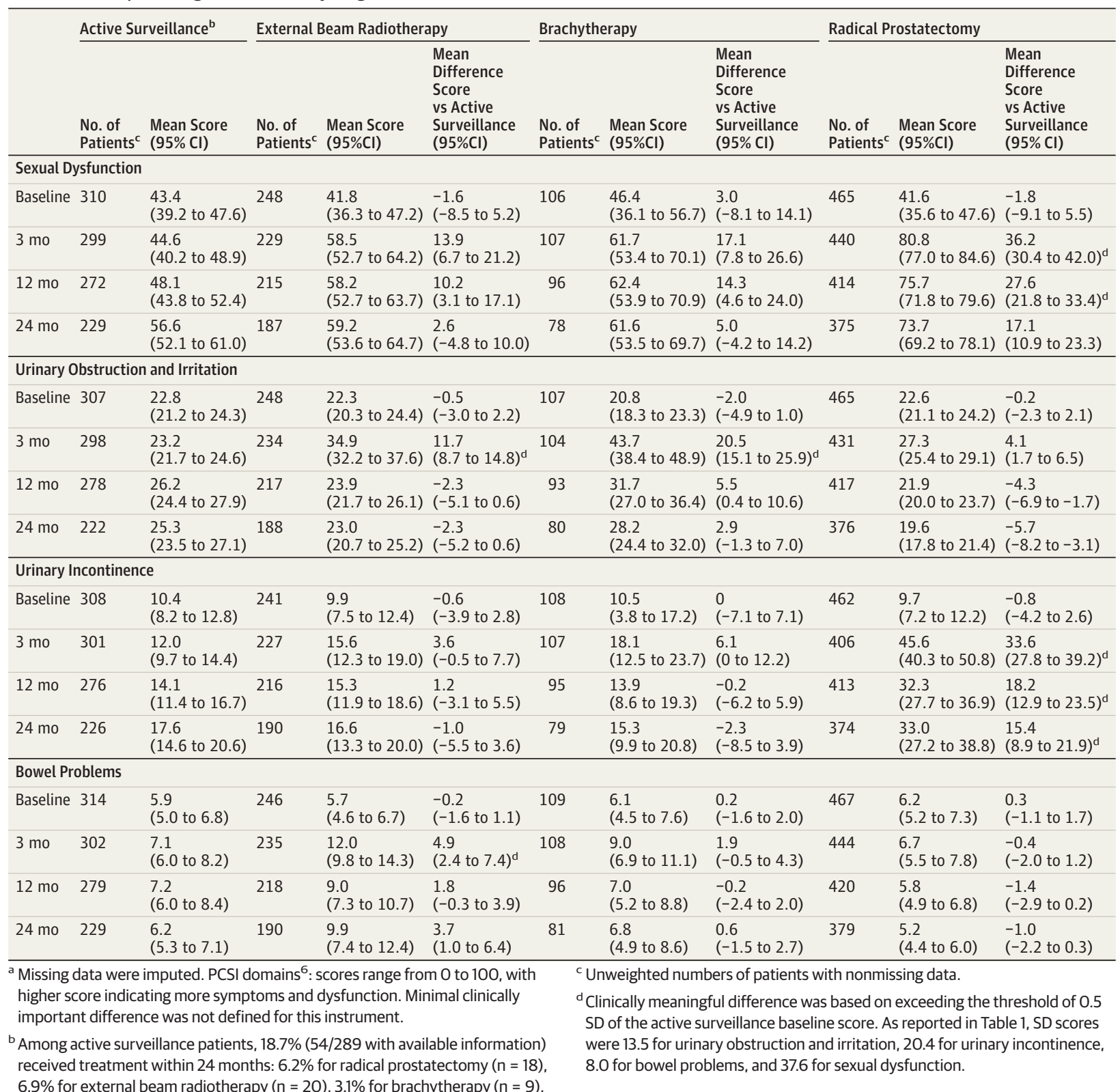

$6.9 \%$ for external beam radiotherapy $(n=20), 3.1 \%$ for brachytherapy $(n=9)$, and $2.4 \%$ for other $(n=7)$.

and brachytherapy had increased sexual dysfunction compared with active surveillance (between-group score difference: external beam radiotherapy vs active surveillance, 13.9 [95\% CI, 6.7-21.2] at 3 months and 10.2 [95\% CI 3.1-17.1] at 12 months; brachytherapy vs active surveillance, 17.1 [95\% CI, 7.8-26.6] at 3 months and 14.3 [95\% CI, 4.6-24.0] at 12 months); however, these differences did not meet the threshold for clinical significance. eTable 3 in the Supplement details sexual dysfunction scores in subgroups of patients who received external beam radiotherapy alone and external beam radiotherapy plus androgen deprivation therapy. The between-group score difference for prostatectomy vs active surveillance met the threshold for clinical significance (36.2 [95\% CI, 30.4-42.0] at 3 months and 27.6 [95\% CI, 21.8-33.4] at 12 months).

Table 3 summarized propensity-weighted results stratifying participants by baseline sexual function level. For men who reported normal baseline sexual function, $57.1 \%$ reported poor function at 24 months after prostatectomy, 27.2\% after external beam radiotherapy, $34.2 \%$ after brachytherapy, and $25.2 \%$ after active surveillance. For men with intermediate baseline function, larger proportions reported poor function at 24 months: $76.9 \%$ after prostatectomy , 55.0\% after external beam radiotherapy, $50.6 \%$ after brachytherapy, and $45.9 \%$ after active 
Table 3. Propensity-Weighted Sexual, Urinary, and Bowel Function at 24 Months by Treatment Type Among Men With Newly Diagnosed Prostate Cancer, Stratified by Baseline Function Level ${ }^{a}$

\begin{tabular}{|c|c|c|c|c|}
\hline & \multirow[b]{2}{*}{ No. of Patients ${ }^{b}$} & \multicolumn{3}{|c|}{ Function Level at 24 Months, $\%(95 \% \mathrm{Cl})$} \\
\hline & & Normal $^{\mathrm{c}}$ & Intermediate $^{\mathrm{d}}$ & Poor $^{\mathrm{e}}$ \\
\hline \multicolumn{5}{|l|}{ Sexual Dysfunction } \\
\hline \multicolumn{5}{|l|}{ Normal baseline level } \\
\hline Active surveillance ${ }^{f}$ & 67 & $44.6(43.3-45.7)$ & $30.3(29.4-31.0)$ & $25.2(24.4-26.1)$ \\
\hline Brachytherapy & 16 & $36.2(34.2-38.5)$ & $29.6(27.2-31.9)$ & $34.2(32.3-36.0)$ \\
\hline Radical prostatectomy & 130 & $9.4(9.1-9.8)$ & $33.5(32.9-34.0)$ & $57.1(56.6-57.6)$ \\
\hline External beam RT & 47 & $27.1(26.2-28.1)$ & $45.7(44.5-46.8)$ & $27.2(26.6-27.9)$ \\
\hline \multicolumn{5}{|l|}{ Intermediate baseline level } \\
\hline Active surveillance $\mathrm{f}^{\mathrm{f}}$ & 64 & $10.2(9.4-10.9)$ & $43.9(43.0-45.0)$ & $45.9(45.0-46.7)$ \\
\hline Brachytherapy & 20 & $2.7(1.5-3.9)$ & $46.7(44.2-49.0)$ & $50.6(48.5-53.0)$ \\
\hline Radical prostatectomy & 111 & $5.1(4.7-5.7)$ & $18.0(17.2-18.8)$ & $76.9(76.1-77.6)$ \\
\hline External beam RT & 46 & $6.6(5.7-7.5)$ & $38.4(37.3-39.7)$ & $55.0(54.0-55.9)$ \\
\hline \multicolumn{5}{|l|}{ Poor baseline level } \\
\hline Active surveillance ${ }^{f}$ & 96 & $2.6(2.2-3.0)$ & $10.1(9.6-10.8)$ & $87.2(86.4-87.9)$ \\
\hline Brachytherapy & 41 & $1.3(0.8-1.7)$ & $20.3(19.5-21.1)$ & $78.5(77.5-79.5)$ \\
\hline Radical prostatectomy & 132 & $1.0(0.9-1.1)$ & $4.3(3.9-4.8)$ & $94.7(94.1-95.2)$ \\
\hline External beam RT & 96 & $1.2(0.9-1.5)$ & $13.8(13.0-14.7)$ & $85.0(84.2-85.9)$ \\
\hline \multicolumn{5}{|c|}{ Urinary Obstruction and Irritation } \\
\hline \multicolumn{5}{|l|}{ Normal baseline level } \\
\hline Active surveillance $\mathrm{f}^{\mathrm{f}}$ & 55 & $42.0(41.1-42.8)$ & $44.8(43.8-45.7)$ & $13.2(12.4-14.1)$ \\
\hline Brachytherapy & 26 & $19.3(17.8-20.9)$ & $56.9(54.8-58.6)$ & $23.9(22.4-25.3)$ \\
\hline Radical prostatectomy & 107 & $52.7(52.3-53.2)$ & $39.1(38.6-39.6)$ & $8.1(7.7-8.6)$ \\
\hline External beam RT & 47 & $48.8(48.0-49.5)$ & $35.8(34.8-36.7)$ & $15.4(14.5-16.4)$ \\
\hline \multicolumn{5}{|l|}{ Intermediate baseline level } \\
\hline Active surveillance ${ }^{f}$ & 94 & $17.0(16.4-17.6)$ & $43.6(42.7-44.5)$ & $39.4(38.6-40.2)$ \\
\hline Brachytherapy & 33 & $18.3(17.0-19.5)$ & $44.1(42.1-46.1)$ & $37.6(36.2-39.0)$ \\
\hline Radical prostatectomy & 131 & $30.9(29.4-32.3)$ & $54.1(52.5-55.9)$ & $15.0(14.2-15.9)$ \\
\hline External beam RT & 69 & $27.6(26.7-28.6)$ & $44.6(43.6-45.5)$ & $27.8(26.8-28.7)$ \\
\hline \multicolumn{5}{|l|}{ Poor baseline level } \\
\hline Active surveillance ${ }^{f}$ & 73 & $6.3(5.6-7.0)$ & $37.0(36.1-38.0)$ & $56.7(55.8-57.7)$ \\
\hline Brachytherapy & 21 & $14.5(13.6-15.4)$ & $33.9(32.1-35.9)$ & $51.5(50.3-52.8)$ \\
\hline Radical prostatectomy & 136 & $24.1(23.3-24.8)$ & $51.6(50.8-52.4)$ & $24.4(23.8-24.9)$ \\
\hline External beam RT & 71 & $9.2(8.5-9.9)$ & $36.7(35.8-37.7)$ & $54.1(53.2-54.8)$ \\
\hline \multicolumn{5}{|l|}{ Urinary Incontinence } \\
\hline \multicolumn{5}{|l|}{ Normal baseline level } \\
\hline Active surveillance ${ }^{f}$ & 169 & $72.7(71.8-73.5)$ & $20.3(19.6-21.0)$ & $7.0(6.7-7.4)$ \\
\hline Brachytherapy & 64 & $64.8(63.7-65.8)$ & $26.2(25.2-27.2)$ & $9.0(8.3-9.7)$ \\
\hline Radical prostatectomy & 288 & $34.3(33.7-34.8)$ & $49.9(49.2-50.6)$ & $15.8(15.4-16.3)$ \\
\hline External beam RT & 130 & $73.0(72.3-73.7)$ & $19.9(19.3-20.5)$ & $7.1(6.6-7.4)$ \\
\hline \multicolumn{5}{|l|}{ Intermediate baseline level } \\
\hline Active surveillance ${ }^{f}$ & 37 & $22.5(21.1-23.7)$ & $51.4(49.4-53.4)$ & $26.1(24.6-27.6)$ \\
\hline Brachytherapy & 13 & $38.1(34.7-41.7)$ & $32.0(27.4-36.6)$ & $29.9(26.7-33.4)$ \\
\hline Radical prostatectomy & 72 & $15.4(14.5-16.2)$ & $67.4(66.4-68.3)$ & $17.3(16.6-17.8)$ \\
\hline External beam RT & 38 & $32.2(31.0-33.6)$ & $52.1(51.0-53.3)$ & $15.7(14.8-16.6)$ \\
\hline \multicolumn{5}{|l|}{ Bowel Problems } \\
\hline \multicolumn{5}{|l|}{ Normal baseline level } \\
\hline Active surveillance $\mathrm{f}^{\mathrm{f}}$ & 96 & $57.2(56.2-58.2)$ & $33.5(32.3-34.8)$ & $9.3(8.7-9.9)$ \\
\hline Brachytherapy & 36 & $46.8(45.1-48.4)$ & $44.4(42.7-46.4)$ & $8.9(7.6-10.5)$ \\
\hline Radical prostatectomy & 172 & $57.4(56.3-58.2)$ & $37.0(36.1-38.0)$ & $5.6(5.2-6.0)$ \\
\hline External beam RT & 76 & $42.7(41.8-43.5)$ & $39.9(39.2-40.5)$ & $17.4(16.7-18.2)$ \\
\hline
\end{tabular}


Table 3. Propensity-Weighted Sexual, Urinary, and Bowel Function at 24 Months by Treatment Type Among Men With Newly Diagnosed Prostate Cancer, Stratified by Baseline Function Level ${ }^{\mathrm{a}}$ (continued)

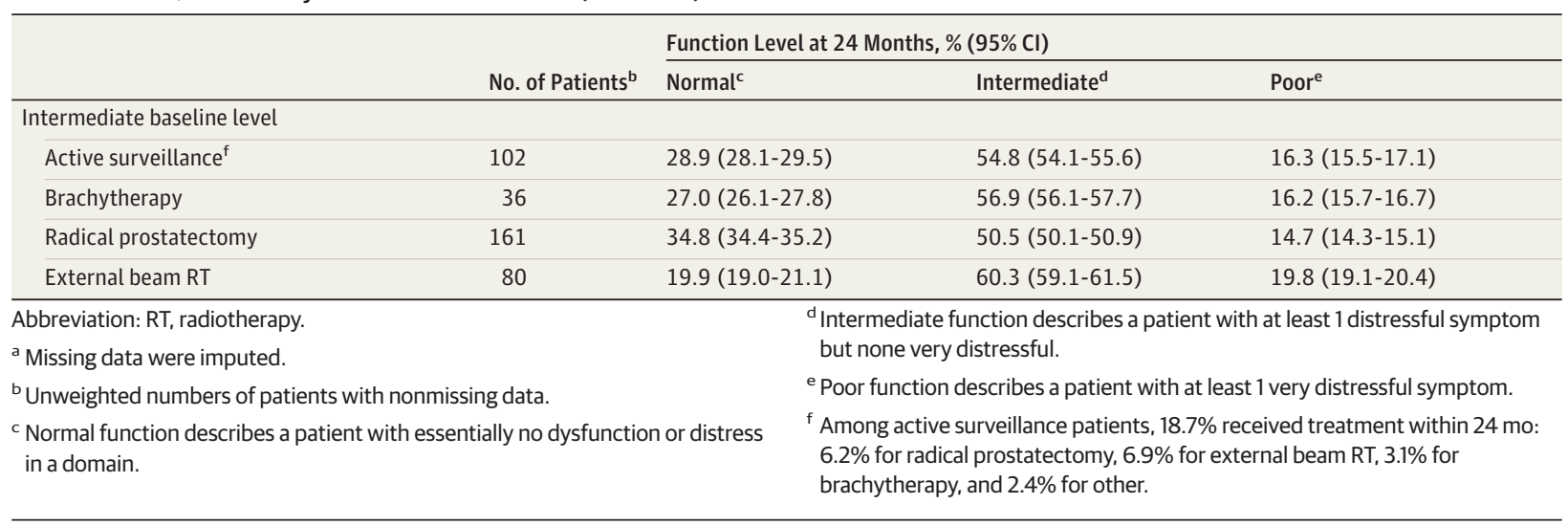

surveillance. Men who reported poor baseline sexual function largely remained with poor function at 24 months.

\section{Urinary Obstruction and Irritation}

At baseline, propensity-weighted scores were 22.8 (95\% CI, 21.2 to 24.3$)$ for active surveillance, 22.3 (95\% CI, 20.3 to 24.4) for external beam radiotherapy, 20.8 (95\% CI, 18.3 to 23.3) for brachytherapy, and 22.6 (95\% CI, 21.1 to 24.2) for prostatectomy (Table 2). Compared with active surveillance, urinary obstruction and irritation scores increased after brachytherapy at 3 and 12 months, but not 24 months (20.5 [95\% CI, 15.1 to 25.9] at 3 months and 5.5 [95\% CI, 0.4 to 10.6] at 12 months), and increased after external beam radiotherapy at 3 months (11.7 [95\% CI, 8.7 to 14.8]). Patients who received prostatectomy reported lower urinary obstruction and irritation compared with active surveillance at 12 and 24 months $(-4.3$ [95\% CI, -6.9 to -1.7 ] at 12 months and -5.7 [95\% CI, -8.2 to -3.1 ] at 24 months). Score differences between brachytherapy and external beam radiotherapy vs active surveillance met the threshold for clinical significance at 3 months.

Table 3 details 24-month outcomes in men with different baseline urinary obstruction and irritation levels. For men at normal baseline function, $52.7 \%$ remained at normal function at 24 months after prostatectomy, $48.8 \%$ after external beam radiotherapy, and $19.3 \%$ after brachytherapy. For men with baseline intermediate function for urinary obstruction and irritation, $18.3 \%$ to $30.9 \%$ improved to normal function after surgical or radiation treatments, whereas $15.0 \%$ to $37.6 \%$ worsened to poor function. At 24 months, most men (75.7\%) with poor baseline function for urinary obstruction and irritation showed improvement after prostatectomy. Among patients in other treatment groups with a poor function level at baseline of urinary obstruction and irritation, 45.9\% improved after external beam radiotherapy, 48.5\% improved after brachytherapy, and 43.3\% improved with initial active surveillance.

\section{Urinary Incontinence}

At baseline, propensity-weighted scores were 10.4 (95\% CI, 8.2-12.8) for active surveillance, 9.9 (95\% CI, 7.5-12.4) for external beam radiotherapy, 10.5 (95\% CI, 3.8-17.2) for brachytherapy, and 9.7 (95\% CI, 7.2-12.2) for prostatectomy (Table 2). Incontinence scores were not significantly different between external beam radiotherapy or brachytherapy vs active surveillance at 3,12, and 24 months. For radical prostatectomy vs active surveillance, incontinence scores increased by 33.6 (95\% CI, 27.8-39.2) at 3 months, 18.2 (95\% CI, 12.923.5) at 12 months, and 15.4 (95\% CI, 8.9-21.9) at 24 months; all of these differences were clinically significant.

For men with normal urinary control at baseline, 34.3\% reported normal control 24 months after prostatectomy, whereas $15.8 \%$ reported poor control (Table 3 ). In other groups, proportions of patients who maintained normal urinary control at 24 months were $73.0 \%$ after external beam radiotherapy, $64.8 \%$ after brachytherapy, and $72.7 \%$ after active surveillance. For men with intermediate urinary control at baseline, $15.7 \%$ to $29.9 \%$ reported poor control at 24 months across the groups. Too few patients had a poor function level of urinary control at baseline $(n=50)$ for meaningful analysis of that subgroup.

\section{Bowel Problems}

At baseline, propensity-weighted scores were 5.9 (95\% CI, 5.06.8) for active surveillance, 5.7 (95\% CI, 4.6-6.7) for external beam radiotherapy, 6.1 (95\% CI, 4.5-7.6) for brachytherapy, and 6.2 (95\% CI, 5.2-7.3) for prostatectomy (Table 2). Compared with active surveillance, external beam radiotherapy increased scores at 3 months (4.9 [95\% CI, 2.4-7.4]) and 24 months (3.7 [95\% CI, 1.0-6.4]). At 3 months, this difference was clinically meaningful. Scores between brachytherapy and prostatectomy vs active surveillance were not significantly different at any time point.

After external beam radiotherapy, $42.7 \%$ of men who reported normal baseline function level also reported a normal function level at 24 months (Table 3). Corresponding proportions were $46.8 \%$ after brachytherapy, $57.4 \%$ after prostatectomy and $57.2 \%$ after active surveillance. In addition, $17.4 \%$ of patients who received external beam radiotherapy who had a normal baseline function level worsened to a poor function level at 24 months, whereas 5.6\% to 9.3\% of patients in other groups worsened to poor function. Too 
few patients were at a poor function level at baseline for meaningful analysis of that subgroup.

\section{Discussion}

Patients with prostate cancer have multiple treatment options and face a confusing decision-making process. Therefore, the comparative effectiveness of contemporary treatment options for localized prostate cancer is a top research priority according to the National Academy of Medicine. ${ }^{1}$ Although contemporary treatments (robotic surgery, intensity-modulated radiotherapy) were developed to reduce treatment-related morbidity, the comparative effectiveness of these treatments vs active surveillance is unknown. A population-based cohort can be used to inform this question by providing timely data from patients who are representative of men with prostate cancer. This study addresses one aspect of this comparative effectiveness question by showing that contemporary treatment options were associated with distinct patterns of QOL changes. These data may help inform men in their treatment decision-making process, and also inform the continued debate regarding prostate cancer screening. The US Preventive Services Task Force, in the current process of revising its screening recommendations, specifically indicated a need to review the harms of different treatment approaches. ${ }^{21}$ This study directly addresses this knowledge gap.

In light of recently published data from the Prostate Testing for Cancer and Treatment (ProtecT) trial, ${ }^{4}$ this study provides new information. This is because the ProtecT trial started in 1999 and used older treatments (3-dimensional radiotherapy, nonrobotic prostatectomy) that may have limited relevance to informing contemporary patients regarding current treatment options, which consist of intensitymodulated radiotherapy, ${ }^{2}$ robotic prostatectomy, ${ }^{22}$ and brachytherapy. ${ }^{23}$ The current study provides QOL outcomes of treatments representative of contemporary choices for patients; the surgical and radiotherapy treatments received by patients in this study ( $86.6 \%$ robotic prostatectomy, $94.8 \%$ intensity-modulated radiotherapy) are reflective of treatment patterns in the United States. ${ }^{2,22}$ With advances in both surgical and radiotherapy technologies, the relative QOL results across treatment groups remain similar as those reported by the ProtecT trial: radical prostatectomy was associated with sexual dysfunction and urinary incontinence, whereas radiotherapy was associated with short-term urinary obstruction and irritation and bowel symptoms.

Active surveillance is an emerging option for men with early prostate cancers, ${ }^{5}$ and served as a natural control group for this study, to help inform the decision for men considering this option vs immediate treatment. This study showed that mean QOL scores for this group remained at baseline levels, until 12 months and after when urinary incontinence and sexual dysfunction scores noticeably increased. This coincides with the timing of guideline-recommended repeat prostate biopsies on active surveillance, with each biopsy expected to detect a proportion of patients with cancer pro- gression necessitating treatment. Although surveillance is the most conservative option, it is notable that mean QOL scores at 24 months were not clinically meaningfully different between this group and active treatment groups in most domains. This observation can be explained by the gradual progression of men on surveillance to treatment over time, whereas men who received immediate treatment experienced gradual improvement in treatment-related symptoms. These findings are consistent with those from the ProtecT trial $^{24}$ and provide information that can be used to help counsel patients-and demonstrates that, on average, active surveillance may be associated with preserved QOL, at least in the first 2 years after diagnosis.

Although QOL research conventionally reports results using mean scores, it is well recognized that score changes are difficult to interpret by patients and physicians. ${ }^{10,12}$ Several approaches have been proposed to address this interpretability challenge, including definition of a minimal clinically important difference or using an arbitrary 0.5-SD change to denote clinically meaningful change. ${ }^{17,18}$ A limitation of the PCSI is that it does not have a defined minimal clinically important difference through an anchor-based method, and therefore we took the latter approach in this study. It is also well established that men with different levels of baseline urinary, bowel, and sexual function likely have different experiences after treatment. ${ }^{9}$ For example, men with no useful erectile function at baseline are unlikely to experience any meaningful positive or negative effect of their sexual function from treatment; therefore, including these men in overall mean score calculations blunts the observed effect (score changes) experienced by other men who have normal baseline function. The PCSI is unique among existing prostate cancer QOL instruments in having defined functional levels, which provides a framework for defining clinically meaningful change and also allows stratification of patients by baseline function. Thus, an additional analysis was performed using QOL levels established by the PCSI instrument to complement the reported mean scores.

Physicians can use these data to provide more individualized counseling of their patients regarding expected outcomes of patients with similar levels of baseline function. For example, among men with normal sexual function at baseline, $57.1 \%$ were estimated to have poor function at 24 months after prostatectomy; $9 \%$ of patients reported preserved normal function, whereas the other one-third experienced declined but still useful sexual function. Men who chose active surveillance had the largest proportion (44.6\%) with preserved normal sexual function at 24 months.

These data also revealed that some QOL aspects can improve after treatment. Older men commonly have benign prostatic hypertrophy with associated urinary obstructive symptoms, which can be improved by prostate cancer treatments. In the group of men who had an intermediate-level of baseline urinary obstruction and irritation symptoms, prostatectomy improved symptoms by 24 months in more patients (30.9\%) than worsened $(15.0 \%)$, and external beam radiotherapy improved and worsened similar proportions. These findings are consistent with prior studies. ${ }^{25,26}$ There are additional possible reasons for some patients experiencing 
improvements in urinary symptoms-including medical management of symptoms and surgical and radiation treatments alleviating symptoms caused by the prostate cancer itself.

There are several strengths of this study. Study design was informed by collaboration with a broad group of stakeholders, ${ }^{20}$ with the goal that this study will provide information relevant to patients and clinicians. This study compared the 4 treatment options most relevant to contemporary patients. However, randomized data suggest that short-term QOL between open vs robotic prostatectomy are not significantly different. ${ }^{27}$ Population-based design facilitated a sociodemographically diverse cohort including patients who chose active surveillance, and not just patients treated at large academic centers. Another strength is that $100 \%$ of patients reported pretreatment QOL without recall, which allows accurate calculations of treatment effect. The study also utilized a consistent methodology for QOL assessment using telephone survey ${ }^{7}$ and time points based on treatment date, with measurements that captured both short-term (3-month) and longer-term changes.

There are several limitations of this study. As an observational study, which is descriptive in nature, there were imbalances in baseline patient characteristics. Propensity weighting was used to minimize these imbalances across groups, but residual confounding is possible and there remained unbalanced disease characteristics across groups with favorable characteristics for active surveillance patients and relatively less favorable characteristics for external beam radiotherapy. Patients were not blinded to treatment received, and patient expectations regarding outcomes from the treatment they received could affect their reporting of QOL. Enrollment in the study was only 57\%, which could have led to a selection bias, although this was not out of the range of other studies. ${ }^{28}$ There was missing data especially with longer follow-up (19\%-29\% at 24-month time point), with differential response rates across groups. In addition, participants were recruited from 1 state. However, they were broadly representative of men with prostate cancer: the median age of NC ProCESS participants (66 years) was similar to prostate cancer patients across the United States, ${ }^{29}$ and the types of radiation ( $94.8 \%$ intensity-modulated radiation therapy) and surgical treatments (86.6\% robotic) received were similar to US patterns of care studies. ${ }^{2,22}$ Also, the Rapid Case Ascertainment system of North Carolina facilitated all participants to be enrolled prior to treatment. This study assessed sexual, urinary, and bowel symptoms, which are most commonly affected after prostate cancer treatments, but did not assess general QOL. In addition, QOL may continue to change more than 2 years after treatment. This analysis included results only through 2 years of follow-up, and further follow-up is ongoing. However, as the ProtecT trial showed, ${ }^{4}$ there may be little change in QOL after 2 years.

\section{Conclusions}

In this cohort of men with localized prostate cancer, each treatment strategy was associated with distinct patterns of adverse effects over 2 years. These findings can be used to promote treatment decisions that incorporate individual preferences.

\section{ARTICLE INFORMATION}

Author Contributions: Dr Chen had full access to all of the data in the study and takes responsibility for the integrity of the data and the accuracy of the data analysis.

Concept and design: Chen, Basak, Meyer, Broughman, Reeve, Anderson.

Acquisition, analysis, or interpretation of data: Chen, Basak, Meyer, Kuo, Carpenter, Agans, Broughman, Reeve, Nielsen, Usinger, Spearman, Walden, Kaleel, Anderson, Stürmer, Godley. Drafting of the manuscript: Chen, Basak, Carpenter, Usinger, Spearman, Kaleel.

Critical revision of the manuscript for important intellectual content: Chen, Basak, Meyer, Kuo, Carpenter, Agans, Broughman, Reeve, Nielsen, Usinger, Walden, Anderson, Stürmer, Godley. Statistical analysis: Chen, Basak, Meyer, Kuo, Broughman, Stürmer.

Obtained funding: Chen, Meyer, Carpenter. Administrative, technical, or material support: Chen, Meyer, Carpenter, Agans, Usinger, Spearman, Walden, Kaleel, Godley.

Supervision: Agans.

No additional contributions: Reeve, Nielsen. Other: Anderson.

Author Affiliations: Department of Radiation Oncology, University of North Carolina at Chapel Hill (Chen, Basak, Broughman); Lineberger Comprehensive Cancer Center, University of North Carolina at Chapel Hill (Chen, Meyer, Kuo, Carpenter, Reeve, Nielsen, Usinger, Spearman, Walden, Kaleel, Stürmer, Godley); Cecil G. Sheps
Center for Health Services Research, University of North Carolina at Chapel Hill (Chen, Nielsen); Department of Epidemiology, Gillings School of Global Public Health, University of North Carolina at Chapel Hill (Meyer, Stürmer); Carolina Survey Research Laboratory, University of North Carolina at Chapel Hill (Agans); Department of Health Policy and Management, University of North Carolina at Chapel Hill (Reeve, Nielsen); Department of Urology, University of North Carolina at Chapel Hill (Nielsen); Patient stakeholder, Prostate Cancer Coalition of North Carolina, Raleigh (Anderson); Division of Hematology and Oncology, Department of Medicine, University of North Carolina at Chapel Hill (Godley).

Conflict of Interest Disclosures: All authors have completed and submitted the ICMJE Form for Disclosure of Potential Conflicts of Interest. Dr Chen reports receiving grants and personal fees from Accuray and personal fees from Astellas and Medivation. Dr Meyer reports having been a member of advisory boards for Merck and ASCO CancerLinQ; these activities were related to large dataset analysis or management and were not related to prostate cancer or this analysis. Dr Nielsen reports receiving grants from the National Institutes of Health (NIH), the American Cancer Society, and personal fees from Urology Care Foundation and Astellas, the American College of Physicians, and Grand Rounds. Dr Stürmer reports receiving grant R01/R56 AG023178 from the National Institute on Aging and grants R01 CA174453 and R01 HL118255 from NIH; salary support from grant UL1TR001111 from North Carolina Translational and Clinical Sciences Institute and the Center for Pharmacoepidemiology (current members: GlaxoSmithKline, UCB BioSciences, Merck); and research support from Amgen and AstraZeneca to his institution; and owning stock in Novartis, Roche, BASF, AstraZeneca, and Novo Nordisk. No other disclosures were reported.

Funding/Support: This article was funded by a Patient-Centered Outcomes Research Institute (PCORI) grant CER-1310-06453 and a contract from the Agency for Healthcare Research and Quality; the Integrated Cancer Information and Surveillance System; University of North Carolina Lineberger Comprehensive Cancer Center; and the University Cancer Research Fund via the state of North Carolina.

Role of the Funder/Sponsor: The sponsor was not involved with the collection, management, analysis, and interpretation of the data; preparation, review, or approval of the manuscript; and decision to submit the manuscript for publication.

Disclaimer: The statements presented in this publication are solely the responsibility of the authors and do not necessarily represent the views of the Patient-Centered Outcomes Research Institute (PCORI), its Board of Governors or Methodology Committee.

Additional Contributions: We thank William Lawrence, MD (PCORI), for his guidance and support of this study. He did not receive compensation for his contribution. 


\section{REFERENCES}

1. Institute of Medicine. Initial National Priorities for Comparative Effectiveness Research. Washington, DC National Academies Press; 2009.

2. Sheets NC, Goldin GH, Meyer AM, et al. Intensity-modulated radiation therapy, proton therapy, or conformal radiation therapy and morbidity and disease control in localized prostate cancer. JAMA. 2012;307(15):1611-1620.

3. Lowrance WT, Eastham JA, Savage C, et al. Contemporary open and robotic radical prostatectomy practice patterns among urologists in the United States. J Urol. 2012;187(6):2087-2092.

4. Donovan JL, Hamdy FC, Lane JA, et al; ProtecT Study Group. Patient-reported outcomes after monitoring, surgery, or radiotherapy for prostate cancer. N Engl J Med. 2016;375(15):1425-1437.

5. Chen RC, Rumble RB, Loblaw DA, et al. Active surveillance for the management of localized prostate cancer (Cancer Care Ontario Guideline): American Society of Clinical Oncology Clinical Practice Guideline Endorsement. J Clin Oncol. 2016; 34(18):2182-2190.

6. Clark JA, Talcott JA. Symptom indexes to assess outcomes of treatment for early prostate cancer. Med Care. 2001;39(10):1118-1130.

7. Sanda MG, Dunn RL, Michalski J, et al. Quality of life and satisfaction with outcome among prostate-cancer survivors. N Engl J Med. 2008;358 (12):1250-1261.

8. Martin NE, Massey L, Stowell C, et al. Defining a standard set of patient-centered outcomes for men with localized prostate cancer. Eur Urol. 2015; 67(3):460-467.

9. Chen RC, Clark JA, Talcott JA. Individualizing quality-of-life outcomes reporting: how localized prostate cancer treatments affect patients with different levels of baseline urinary, bowel, and sexual function. J Clin Oncol. 2009;27(24):3916-3922.

10. Chen RC, Chang P, Vetter RJ, et al. Recommended patient-reported core set of symptoms to measure in prostate cancer treatment trials. J Natl Cancer Inst. 2014;106(7): dju132.
11. Mushlin Al, Ghomrawi H. Health care reform and the need for comparative-effectiveness research. N Engl J Med. 2010;362(3):e6.

12. Talcott JA, Clark JA, Manola J, Mitchell SP. Bringing prostate cancer quality of life research back to the bedside: translating numbers into a format that patients can understand. J Urol. 2006 176(4 pt 1):1558-1563; discussion 1563-1564.

13. van Buuren S. Multiple imputation of discrete and continuous data by fully conditional specification. Stat Methods Med Res. 2007;16(3): 219-242.

14. Stürmer T, Wyss R, Glynn RJ, Brookhart MA Propensity scores for confounder adjustment when assessing the effects of medical interventions using nonexperimental study designs. J Intern Med. 2014;275(6):570-580.

15. Austin PC, Stuart EA. Moving towards best practice when using inverse probability of treatment weighting (IPTW) using the propensity score to estimate causal treatment effects in observational studies. Stat Med. 2015;34(28) 3661-3679.

16. White IR, Royston P, Wood AM. Multiple imputation using chained equations: issues and guidance for practice. Stat Med. 2011;30(4):377-399.

17. Norman GR, Sloan JA, Wyrwich KW. Interpretation of changes in health-related quality of life: the remarkable universality of half a standard deviation. Med Care. 2003;41(5):582-592.

18. Norman GR, Sloan JA, Wyrwich KW. The truly remarkable universality of half a standard deviation: confirmation through another look. Expert Rev Pharmacoecon Outcomes Res. 2004;4(5):581-585.

19. Wright JG. Interpreting health-related quality of life scores: the simple rule of 7 may not be so simple. Med Care. 2003;41(5):597-598.

20. Chen RC, Carpenter WR, Kim M, et al. Design of the North Carolina Prostate Cancer Comparative Effectiveness and Survivorship Study (NC ProCESS). J Comp Eff Res. 2015;4(1):3-9.

21. US Preventive Services Task Force. Final research plan for prostate cancer: screening. http://www.uspreventiveservicestaskforce.org /Page/Document/final-research-plan/prostate -cancer-screening1. Accessed September 1, 2016.

22. Oberlin DT, Flum AS, Lai JD, Meeks JJ. The effect of minimally invasive prostatectomy on practice patterns of American urologists. Urol Oncol. 2016;34(6):255.e1-255.e5.

23. Safdieh J, Wong A, Weiner JP, Schwartz D, Schreiber D. Utilization of prostate brachytherapy for low risk prostate cancer: is the decline overstated? J Contemp Brachytherapy. 2016;8(4): 289-293.

24. Wilt TJ. The Prostate Cancer Intervention vs Observation Trial: VA/NCI/AHRQ Cooperative Studies Program \#407 (PIVOT): design and baseline results of a randomized controlled trial comparing radical prostatectomy with watchful waiting for men with clinically localized prostate cancer. J Nat Cancer Inst Monogr. 2012;2012(45):184-190.

25. Steineck G, Helgesen F, Adolfsson J, et al; Scandinavian Prostatic Cancer Group Study Number 4. Quality of life after radical prostatectomy or watchful waiting. N Engl J Med. 2002;347(11):790-796.

26. Chang $P$, Regan MM, Ferrer $M$, et al; PROST-QA Consortium. Relief of urinary symptom burden after primary prostate cancer treatment. J Urol. 2017;197 (2):376-384.

27. Yaxley JW, Coughlin GD, Chambers SK, et al. Robot-assisted laparoscopic prostatectomy versus open radical retropubic prostatectomy: early outcomes from a randomised controlled phase 3 study. Lancet. 2016;388(10049):1057-1066.

28. Potosky AL, Harlan LC, Stanford JL, et al. Prostate cancer practice patterns and quality of life the Prostate Cancer Outcomes Study. J Natl Cancer Inst. 1999;91(20):1719-1724.

29. Miller KD, Siegel RL, Lin CC, et al. Cance treatment and survivorship statistics, 2016. CA Cancer J Clin. 2016;66(4):271-289. 